\title{
Process and Product Innovation by a Multiproduct Monopolist: A Dynamic Approach
}

\author{
Luca Lambertini*and Andrea Mantovani ${ }^{\dagger}$
}

October 18, 2005

\begin{abstract}
We model the optimal behaviour of a multiproduct monopolist investing both in process and in product innovation in a dynamic setting. Product innovation reduces the degree of substitutability between any two varieties. First, we find that $R \& D$ efforts increase in both directions as the number of varieties grows. Second, we characterise the relative intensity of $R \& D$ activities according to the reservation price and the interaction between the number of varieties and the degree of product differentiation. Finally, we show the existence of complementarity within the R\&D portfolio, i.e., decreasing marginal production cost prompts for an analogous reduction of product substitutability, and conversely.
\end{abstract}

JEL classification: D42, D92, O31.

Keywords: product innovation, process innovation, complementarity

*Department of Economics, University of Bologna, Strada Maggiore 45, I-40125 Bologna, Italy, lamberti@spbo.unibo.it; and ENCORE, Faculty of Economics \& Econometrics, University of Amsterdam, Roetersstraat 11, WB1018 Amsterdam, The Netherlands.

${ }^{\dagger}$ Department of Economics, University of Bologna, Strada Maggiore 45, I-40125 Bologna, Italy, mantovan@spbo.unibo.it 


\section{Introduction}

Casual observation suggests that firms activate $R \& D$ portfolios including several projects aimed at either product or process innovation. ${ }^{1}$ In the vast majority of cases, the twin issues of process and product innovation are treated separately. A turning point in the approach to the analysis of the optimal design of $R \& D$ activities along several dimensions at the same time can be perhaps identified in the attention recently devoted to the role of complementarities (Vives, 1990; Milgrom and Roberts, 1990; Amir, 1996). This toolkit has been used to investigate the presence of complementarities within $\mathrm{R} \& \mathrm{D}$ portfolios in monopoly (Athey and Schmutzler, 1995; Lambertini and Orsini, 2000; Lambertini, 2003, 2004; Lin, 2004; Mantovani, 2005) or oligopoly (Bonanno and Haworth, 1998; Lin and Saggi, 2002; Rosenkranz, 2003). The bottom line of this stream of research is that R\&D efforts in each direction boosts the firms' incentive to carry out analogous effort in the other direction. Others have stressed the difference between innovations and improvements, i.e., between technological breakthroughs and engineering refinements (see Doraszelski, 2004). ${ }^{2}$

However, notwithstanding the fact that $R \& D$ is an inherently dynamic feature of a firm's activities, most of the aforementioned contributions are in fact of a static nature. Accordingly, it would be desirable to characterise the interplay between process and product innovation in a properly dynamic setup. To this aim, we model the optimal behaviour of a multiproduct monopolist investing both in process and in product innovation. By process innovation we mean that the firm invests in order to reduce the marginal produc-

\footnotetext{
${ }^{1}$ Two relevant examples are the contributions of Bhattacharya and Mookherjee (1986) and Dasgupta and Maskin (1987).

${ }^{2} \mathrm{~A}$ related literature describes the dynamics of product and process innovation in connection with technology life cycle. The common view emerging from this strand is that product innovation necessarily precedes process innovation. See Abernathy and Utterback (1975, 1982), Klepper (1996) and Adner and Levinthal (2001).
} 
tion cost which is assumed to be the same for all varieties, while by product innovation we mean that the firm wants to increase the degree of differentiation between any two varieties. This problem is built up as an optimal control model where the marginal cost and the degree of differentiation (or substitutability) are state variables, with the firm controlling output and R\&D levels at any time over an infinite horizon.

The main results of our analysis can be outlined as follows. We identify the conditions ensuring the existence of a unique steady state equilibrium, which is a saddle point. The economic features of the model, describing the qualitative properties of the optimal behaviour of the firm, are as follows. First, R\&D efforts intensify in both directions as the number of varieties increases. This is intuitively due to a cannibalization effect taking place among product varieties as the product space become more and more crowded. In order to safeguard its profitability, the monopolist has to invest higher amounts of resources to lower both variable production costs and substitutability. The second result has to do with the relative intensity of $R \& D$ efforts. To this regard, we prove that, if the reservation price is sufficiently low, the firm finds it convenient to devote a larger amount of resources to process rather than product innovation irrespectively of the extent of the product range and the associated level of differentiation. This is driven by the fact that the interval between the reservation price and marginal cost is too narrow. If instead the reservation price is high enough, then the relative intensity of $R \& D$ efforts is determined by the interaction between the number of varieties and the degree of differentiation, i.e., the cannibalization problem. If differentiation is low, then product $\mathrm{R} \& \mathrm{D}$ draws a larger amount of resources than process $\mathrm{R} \& \mathrm{D}$. Conversely, if differentiation is high, the allocation of resources over the $\mathrm{R} \& \mathrm{D}$ portfolio also depends upon the number of varieties, so that the firm may indeed invest more in process rather than in product innovation. The third result holds irrespective of the demand level and the extension of the product range: we show that there always exists complementarity within the R\&D portfolio, i.e., decreasing marginal production cost prompts for an analogous reduction of product substitutability, 
and conversely. This is true both along the optimal paths to the steady state and in steady state.

The outline of the paper is as follows. Section 2 illustrates the basics of the model. the equilibrium analysis is in section 3 . Section 4 concludes the paper.

\section{The Model}

We build an optimal control problem over continuous time $t \in[0, \infty)$, where at any instant a multiproduct monopolist chooses the quantity level for each of $N$ product varieties and the investment level in product and process innovation. Process innovation is formalized as a reduction of the unit cost of production, while product innovation affects product substitutability as perceived by consumers.

As in Spence (1976) and Singh and Vives (1984), the representative consumer's instantaneous utility function is defined in terms of the consumption of $N$ differentiated goods and the numeraire good $m>0$, and is given by:

$$
u(\mathbf{q}(t), m)=a \sum_{i=1}^{N} q_{i}(t)-\frac{1}{2}\left(\sum_{i=1}^{N}\left[q_{i}(t)\right]^{2}+s(t) \sum_{i=1}^{N} \sum_{j \neq i} q_{i}(t) q_{j}(t)\right)+m,
$$

where $\mathbf{q}(t) \equiv\left(q_{1}(t), q_{2}(t) \ldots q_{N}(t)\right)$ is the vector of quantities consumed at any instant $t, a$ is a positive parameter and $s(t) \in[0,1]$. The state variable $s(t)$ represents the degree of substitutability between any two products. If $s(t)=1$, products are completely homogeneous. If instead $s(t)=0$, products are independent and variety $i$ 's price is unaffected by the supply of any other variety. Constrained utility maximization for any given price vector $\mathbf{p}(t) \equiv\left(p_{1}(t), p_{2}(t) \ldots p_{N}(t)\right)$ gives rise to the following demand system:

$$
p_{i}(t)=a-q_{i}(t)-s(t) \sum_{j \neq i} q_{j}(t) \quad \forall i \neq j
$$

where at any time $t$, the production of output level $q_{i}(t)$ involves a linear cost function, so that the instantaneous cost function of the monopolist is $C(\mathbf{q}(t))=c(t) \sum_{i=1}^{N} q_{i}(t)$, 
where $c(t)$ is the unit cost of production common to all varieties.

We assume that product differentiation can be affected by $R \& D$ investment in product innovation. The dynamics associated to product innovation is described by the kinematic equation:

$$
\frac{d s(t)}{d t} \equiv \dot{s}=s(t)[-x(t)+\delta]
$$

where $x(t)$ represents the effort made by the firm at time $t$ in order to increase product differentiation through a reduction of $s(t) .{ }^{3}$ Parameter $\delta \in[0,1]$ indicates the depreciation rate due to ageing of technology. Equation (3) can be rewritten as follows:

$$
\frac{\dot{s}}{s(t)}=-x(t)+\delta
$$

so as to highlight that the rate of change of product substitutability over time is linear in the instantaneous investment efforts.

Moreover, the firm invests in process innovation; as a consequence, unit cost $c(t)$ evolves over time as described by the following:

$$
\frac{d c(t)}{d t} \equiv \dot{c}=c(t)[-k(t)+\eta]
$$

where $k(t)$ is the $\mathrm{R} \& \mathrm{D}$ effort for process innovation. Parameter $\eta \in[0,1]$ is the depreciation rate. The rate of change of unit cost over time is linear in the instantaneous investment effort, given that:

$$
\frac{\dot{c}}{c(t)}=-k(t)+\eta
$$

Equation (5) is indeed a dynamic version of the linear R\&D technology employed by Lambertini (2003, 2004) and Lin (2004).

The instantaneous cost of investing in product innovation and in process innovation is respectively given by $C(x(t))=\gamma[x(t)]^{2}$ and $C(k(t))=\beta[k(t)]^{2}$, where $\gamma$ and $\beta$ are

\footnotetext{
${ }^{3}$ The idea that $s$ depends upon firms' investment decisions has been investigated in static models by Harrington (1995), Lambertini and Rossini (1998) and Lambertini, Poddar and Sasaki (1998). Recent contributions apply this idea to differential games, e.g. Cellini and Lambertini (2002 and 2004).
} 
positive parameters. Hence, investing in both types of $R \& D$ implies decreasing returns to innovative activity.

Gross profits from sales are $\psi_{i}(t)=\left[a-q_{i}(t)-s(t) \sum_{j \neq i} q_{j}(t)-c(t)\right] q_{i}(t)$ for each variety $i=1,2, \ldots N$. Instantaneous profits are given by:

$$
\pi(t)=\sum_{i=1}^{N}\left[a-q_{i}(t)-s(t) \sum_{j \neq i} q_{j}(t)-c(t)\right] q_{i}(t)-\gamma[x(t)]^{2}-\beta[k(t)]^{2}
$$

The monopolist aims at maximizing the discounted profit flow:

$$
\Pi(t)=\int_{0}^{\infty} \pi(t) e^{-\rho t} d t
$$

w.r.t. R\&D efforts $x(t), k(t)$ and the vector of market variables $\mathbf{q}(t)$, under the constraint given by the state dynamics (3) and (5). Therefore the problem features $N+2$ controls and two states. The discount rate $\rho>0$ is assumed to be constant. The solution of the monopolist's maximization problem is illustrated in the next section.

\section{Optimal Process and Product Innovation}

Using the state dynamics (3) and (5) and the expression of the instantaneous profits (7), we may write the current value Hamiltonian function of the firm as follows:

$$
\mathcal{H}(t)=e^{-\rho t}\left[\pi(t)+\lambda_{s}(t) \dot{s}+\lambda_{c}(t) \dot{c}\right]
$$

where $\lambda_{s}(t)=\mu_{s}(t) e^{\rho t}, \lambda_{c}(t)=\mu_{c}(t) e^{\rho t}, \mu_{s}(t)$ and $\mu_{c}(t)$ being the co-state variables associated to $s(t)$ and $c(t)$, respectively. The first order conditions (FOCs) on controls are: ${ }^{4}$

$\frac{\partial \mathcal{H}(t)}{\partial q_{i}(t)}=a-c(t)-2\left(q_{i}(t)+s(t) \sum_{j \neq i} q_{j}(t)\right)=0 \Rightarrow q_{i}^{*}(t)=\frac{a-c(t)-2 s(t) \sum_{j \neq i} q_{j}(t)}{2}$

\footnotetext{
${ }^{4}$ For the sake of brevity, in the remainder we omit the indication of exponential discounting. Moreover, second order conditions are also omitted throughout the paper. They are always met, as it can be verified using different methods (see Mangasarian, 1966; Arrow, 1968; Stalford and Leitmann, 1973).
} 


$$
\begin{aligned}
& \frac{\partial \mathcal{H}(t)}{\partial x(t)}=-2 \gamma x(t)-\lambda_{s}(t) s(t)=0 \Rightarrow x^{*}(t)=-\frac{\lambda_{s}(t) s(t)}{2 \gamma} \\
& \frac{\partial \mathcal{H}(t)}{\partial k(t)}=-2 \beta k(t)-\lambda_{c}(t) c(t)=0 \Rightarrow k^{*}(t)=-\frac{\lambda_{c}(t) c(t)}{2 \beta}
\end{aligned}
$$

The corresponding co-state equations are:

$$
\begin{gathered}
-\frac{\partial \mathcal{H}(t)}{\partial s(t)}=\dot{\lambda}_{s}-\rho \lambda_{s}(t) \Rightarrow \\
\dot{\lambda}_{s}=\sum_{i=1}^{N} q_{i}(t) \sum_{j \neq i} q_{j}(t)-\lambda_{s}(t)[\delta-\rho-x(t)] \\
-\frac{\partial \mathcal{H}(t)}{\partial c(t)}=\dot{\lambda}_{c}-\rho \lambda_{c}(t) \Leftrightarrow \dot{\lambda}_{c}=\sum_{i=1}^{N} q_{i}(t)-\lambda_{c}(t)[\eta-\rho-k(t)]
\end{gathered}
$$

along with the transversality condition:

$$
\lim _{t \rightarrow \infty} \mu_{s}(t) s(t)=0 ; \lim _{t \rightarrow \infty} \mu_{c}(t) c(t)=0 .
$$

Now, from (11) we have:

$$
\lambda_{s}(t)=-\frac{2 \gamma x(t)}{s(t)}
$$

as well as the expression of the optimal $x(t)$, which can be differentiated w.r.t. $t$ :

$$
\dot{x}=-\frac{1}{2 \gamma}[\dot{\lambda} s(t)+\lambda(t) \dot{s}] .
$$

Following an analogous procedure with (12), we obtain:

$$
\lambda_{c}(t)=-\frac{2 \beta k(t)}{c(t)}
$$

and

$$
\dot{k}=-\frac{1}{2 \beta}\left[\dot{\lambda}_{c} c(t)+\lambda_{c}(t) \dot{c}\right] .
$$

For intuitive reasons, without loss of generality we can impose symmetry on output levels, so that $q_{i}(t)=q(t)$ for all $i=1,2, \ldots N$. Then, using (3) and (5), together with (13-14) as well as (16) and (18), we can rewrite (17) and (19) as follows:

$$
\dot{x}=\rho x-\frac{N(N-1)[q(t)]^{2} s(t)}{2 \gamma}
$$




$$
\dot{k}=\rho k-\frac{N q(t) c(t)}{2 \beta} .
$$

The next step consists in solving (10) w.r.t. $q(t)$ :

$$
q^{*}(t)=\frac{a-c(t)}{2[1+s(t)(N-1)]}
$$

which can be substituted into (20-21):

$$
\begin{gathered}
\dot{x}=\rho x-\frac{N(N-1)[a-c(t)]^{2} s(t)}{8 \gamma[1+s(t)(N-1)]^{2}} \\
\dot{k}=\rho k-\frac{N[a-c(t)] c(t)}{4 \beta[1+s(t)(N-1)]} .
\end{gathered}
$$

On the basis of the above differential equations describing the rates of change of $R \& D$ controls, we find a relevant property:

Proposition 1 The paths of RESD investments are characterised by strategic complementarity at any point in time.

Proof. Examine the following partial derivatives:

$$
\begin{aligned}
\frac{\partial \dot{x}}{\partial c(t)} & =\frac{N(N-1)[a-c(t)] s(t)}{32\left\{\gamma[1+s(t)(N-1)]^{2}\right\}^{2}}>0 \\
\frac{\partial \dot{k}}{\partial s(t)} & =\frac{N(N-1)[a-c(t)] c(t)}{4 \beta[1+s(t)(N-1)]^{2}}>0
\end{aligned}
$$

These suffice to prove that any decrease in marginal cost (resp. product substitutability) triggers an increase in the instantaneous rate of change of the $\mathrm{R} \& \mathrm{D}$ activity for product (resp., process) innovation. This proves the claim.

With reference to the result stated in Proposition 1, which reveals the existence of dynamic strategic complementarity between R\&D activities, one may wonder whether this property characterises the equilibrium efforts as well. This is going to be verified below. 
Now, imposing the stationarity conditions $\dot{x}=0$ and $\dot{k}=0$, we find the optimal investment levels in terms of states, the number of products and the parameters of the model:

$$
x^{*}=\frac{N(N-1)[a-c(t)]^{2} s(t)}{8 \gamma \rho[1+s(t)(N-1)]^{2}} ; k^{*}=\frac{N[a-c(t)] c(t)}{4 \beta \rho[1+s(t)(N-1)]} .
$$

A natural question that springs to mind is whether the firm has a higher incentive to invest in process or product $\mathrm{R} \& \mathrm{D}$, and under what circumstances either effort is larger than the other. To this regard, we can prove:

Proposition 2 Consider optimal investments $x^{*}$ and $k^{*}$. The following holds:

- suppose $a \in\left(c, \frac{(\beta+2 \gamma)}{\beta} c\right]$. If so, then $k^{*}>x^{*}$ always;

- now suppose instead $a>\frac{(\beta+2 \gamma)}{\beta} c$. In such a case, then: (i) if $s \in[0,1 / 2)$, then $k^{*}>x^{*}$ for all $N \in\left[1, \frac{2 \gamma c(1-s)+\beta s(a-c)}{[\beta(a-c)+2 \gamma c] s}\right)$; and $k^{*}<x^{*}$ for all $N>$ $\frac{2 \gamma c(1-s)+\beta s(a-c)}{[\beta(a-c)+2 \gamma c] s}$; (ii) if $s \in[1 / 2,1]$, then $k^{*}<x^{*}$ for all $N \geq 1$.

Proof. From (26), we have

$$
x^{*}>k^{*} \Leftrightarrow N[\beta(a-c)+2 \gamma c] s>2 \gamma c(1-s)+\beta s(a-c) .
$$

Note that the r.h.s. is always positive, while the l.h.s. may take either sign. If $a<$ $(\beta+2 \gamma) c / \beta$, which is an admissible case as $(\beta+2 \gamma) / \beta>1$, then surely $x^{*}<k^{*}$. Otherwise, the sign of $x^{*}-k^{*}$ depends upon the value of $N$ and $s$. In particular, provided $a>(\beta+2 \gamma) c / \beta$, we have $x^{*}>k^{*}$ if

$$
N>\frac{2 \gamma c(1-s)+\beta s(a-c)}{[\beta(a-c)+2 \gamma c] s},
$$

with

$$
\frac{2 \gamma c(1-s)+\beta s(a-c)}{[\beta(a-c)+2 \gamma c] s}>1 \forall s \in[0,1 / 2)
$$


Therefore, if $s \in[1 / 2,1]$,

$$
\frac{2 \gamma c(1-s)+\beta s(a-c)}{[\beta(a-c)+2 \gamma c] s}<1
$$

and, as a consequence, $k^{*}<x^{*}$ for all $N \geq 1$.

The above result can be given the following intuitive explanation. When the reservation price is comparatively low, then the monopolist finds it convenient to devote a larger amount of resources to process rather than product innovation for any level of both $N$ and $s$, because the distance between the reservation price and marginal cost is too narrow. Otherwise, if $a$ is high enough, the relative intensity of $R \& D$ efforts depends upon the interplay between the extension of the product range and the degree of substitutability between any two varieties. This interaction accounts for a crowding effect in the product space which is intimately connected to cannibalization. When $s$ is large, the lack of differentiation calls for a higher investment in this direction. When instead $s$ is already low enough, then the relative weight of process and product innovation in the firm's R\&D portfolio is also determined by the number of varieties being supplied. If such a number is larger than the critical threshold highlighted in the proposition, then cannibalization drives the result that $x^{*}>k^{*}$.

The expressions (26) can be used to carry out comparative statics on optimal R\&D efforts. In particular, the following properties can be easily singled out:

$$
\frac{\partial x^{*}}{\partial s(t)} \propto-[s(t)(N-1)-1]
$$

so that $\partial x^{*} / \partial s(t)>0$ iff $s(t)<1 /(N-1) \equiv \widehat{s}$. Note that $\widehat{s} \in[0,1]$ iff $N \geq 2$. Moreover, $\partial \widehat{s} / \partial N<0$ always, with $\lim _{N \rightarrow \infty} \widehat{s}=0$. As to the investment in process innovation, $\partial k^{*} / \partial s(t)<0$ always. Therefore, we may claim:

Proposition 3 The interval wherein $\partial x^{*} / \partial s(t)>0$ is monotonically decreasing in the number of product varieties. The incentive to carry out process innovation increases monotonically in the degree of product differentiation. 
What the Proposition says is that the $R \& D$ effort for product innovation is increasing in the level of product substitutability if the latter is below a threshold value which, in turn, is negatively correlated with the number of varieties. The intuition can be found in the balance between three factors. The first is that, all else equal, if $s(t)$ is large, then the firm has a clear-cut incentive to reduce it by increasing $x^{*}$. The second is that the $\mathrm{R} \& \mathrm{D}$ effort involves a cost which is quadratic in the effort itself. The third factor has to do with internal fund raising to finance $R \& D$, i.e., the fact that innovation expenditure is paid for by using gross profits obtained from sales, which are decreasing in the degree of substitutability. ${ }^{5}$ The first factor clearly exerts a positive effect on $x^{*}$, while the opposite holds for the second and the third one. If the cost and sales effects overcome the pure incentive to invest in product differentiation, then, overall, $x^{*}$ slopes downward as $s(t)$ becomes larger. The second claim in Proposition 3 reveals that there exists complementarity between process and product innovation. An intuitive reason is that any increase in product differentiation involves larger internal funding to be used to finance also $R \& D$ in the other direction.

Now we turn to examine the effect of a change in marginal production cost on the $\mathrm{R} \& \mathrm{D}$ incentives measured by (26). First, note that $\partial k^{*} / \partial c(t) \propto a-2 c(t)>0$ for all $c(t) \in[0, a / 2)$. Then, $\partial x^{*} / \partial c(t) \propto-[a-c(t)]<0$ always. These partial derivatives yield:

Proposition 4 The RED effort for process innovation is concave and single-peaked w.r.t. marginal production cost. The incentive to carry out product innovation decreases monotonically in the level of the marginal production cost.

\footnotetext{
${ }^{5}$ Indeed, sales profits for each variety are given by the expression

$$
\psi_{i}\left(\mathbf{q}^{*}\right)=\frac{[a-c(t)]^{2}}{4[1+s(t)(N-1)]}
$$

which is clearly decreasing in $s(t)$.
} 
Obviously, the second part of the above Proposition confirms the presence of complementarity between the two $R \& D$ activities. This result is qualitatively close to previous research carried out in static models of product and process innovation (Lin and Saggi, 2002; Lambertini 2003, inter alia), although, in the present case, complementarity between the two activities is the outcome of dynamic optimization rather than the backward induction method typically employed in multistage static games. In other words, the presence of complementarity at equilibrium, highlighted in Proposition 3-4, is nothing but the natural outcome of the dynamic complementarity illustrated in Proposition 1.

The next step consists in analysing what happens to optimal investments as a result of a change in the extent of the product range:

$$
\begin{gathered}
\frac{\partial x^{*}}{\partial N}=-\frac{[a-c(t)]^{2}[1-s(t)-N(2-s(t))] s(t)}{8 \gamma \rho[1+s(t)(N-1)]^{3}}>0 \\
\frac{\partial k^{*}}{\partial N}=\frac{[a-c(t)] c(t)[1-s(t)]}{4 \beta \rho[1+s(t)(N-1)]^{2}}>0
\end{gathered}
$$

Both features can be explained on the basis of the cannibalization effect obtaining as $N$ increases, whereby introducing any additional product variety generates a negative externality on the existing ones, whose profitability declines as a result of a congestion of the product space. ${ }^{6}$ Accordingly, we can state:

Proposition 5 Any expansion of the product range calls for more intense R\&D efforts in both directions.

Using (26) and imposing stationarity in turn on state equations, we find the following expressions for the state variables:

$$
s^{*}(c)=\frac{N(a-c)^{2}-16 \delta \gamma \rho-(a-c) \sqrt{N\left[N(a-c)^{2}-32 \delta \gamma \rho\right]}}{16 \delta \gamma \rho(N-1)}
$$

\footnotetext{
${ }^{6}$ Also $N$ could be endogenously determined as a state variable requiring its own investment. For a (static) model where the monopolist invests to increase the number of varieties while taking as given the degree of substitutability, see Lambertini (2003, 2004) and Lin (2004).
} 


$$
c^{*}(s)=\frac{a N-\sqrt{N\left[a^{2} N-16 \beta \eta \rho(1+s(N-1))\right]}}{2 N}
$$

Provided that $s^{*}(c)$ and $c^{*}(s)$ belong to $\mathbb{R}$, then also $s^{*}(c) \in[0,1]$ and $c^{*}(s) \in[0, a]$. However, note that

$$
\begin{aligned}
& s^{*}(c) \in \mathbb{R} \text { iff } \rho<\frac{N(a-c)^{2}}{32 \delta \gamma} \\
& c^{*}(s) \in \mathbb{R} \text { iff } \rho<\frac{a^{2} N}{16 \beta \eta(1+s(N-1))}
\end{aligned}
$$

Therefore:

\section{Lemma 6 If}

$$
\rho \in\left[0, \min \left\{\frac{N(a-c)^{2}}{32 \delta \gamma}, \frac{a^{2} N}{16 \beta \eta(1+s(N-1))}\right\}\right)
$$

then there exists an internal solutions along both dimensions of the monopolist's RED activity.

Finally, we come to the evaluation of the stability properties of the model. Given that the Jacobian matrix is $4 \times 4$, one cannot draw the phase diagram. The Jacobian matrix is:

$$
J^{*}=\left[\begin{array}{cccc}
\frac{\partial \dot{s}}{\partial s}=\delta-x & \frac{\partial \dot{s}}{\partial x}-s & \frac{\partial \dot{s}}{\partial c}=0 & \frac{\partial \dot{s}}{\partial k}=0 \\
\frac{\partial \dot{x}}{\partial s}=\frac{(a-c)^{2}(N-1)[s(N-1)-1]}{8 \gamma[1+s(N-1)]^{3}} & \frac{\partial \dot{x}}{\partial x}=\rho & \frac{\partial \dot{x}}{\partial c}=\frac{(a-c) N(N-1) s}{4 \gamma[1+s(N-1)]^{2}} & \frac{\partial \dot{x}}{\partial k}=0 \\
\frac{\partial \dot{c}}{\partial s}=0 & \frac{\partial \dot{c}}{\partial x}=0 & \frac{\partial \dot{c}}{\partial c}=\eta-k & \frac{\partial \dot{c}}{\partial k}=-c \\
\frac{\partial \dot{k}}{\partial s}=\frac{c(a-c) N(N-1)}{4 \beta[1+s(N-1)]^{2}} & \frac{\partial \dot{k}}{\partial x}=0 & \frac{\partial \dot{k}}{\partial c}=-\frac{(a-2 c) N}{4 \beta[1+s(N-1)]} & \frac{\partial \dot{k}}{\partial k}=\rho
\end{array}\right]
$$

whose characteristic equations yields four eigenvalues. Unfortunately, assessing the sign of such eigenvalues analytically is not feasible as their expressions are cumbersome. Moreover, we cannot obtain the explicit solutions for $c^{*}$ and $s^{*}$ as a function of parameters 
only, as the system

$$
\begin{aligned}
& c-c^{*}(s)=0 \\
& s-s^{*}(c)=0
\end{aligned}
$$

is made up by equations whose degree is higher than four.

However, we may resort to numerical calculations, which can be performed as follows. We use the solutions:

$$
x^{*}=\delta ; k^{*}=\eta
$$

and set the numerical values of parameters:

$$
a=1 ; \beta=\gamma=\frac{1}{2} ; \delta=\eta=\rho=\frac{1}{20} ; N=3
$$

Then, we may (i) solve the system (38) numerically, and (ii) compute the eigenvalues $\left\{\zeta_{1}, \zeta_{2}, \zeta_{3}, \zeta_{4}\right\}$ of $J^{*}$. Given (40), we have:

$$
\begin{gathered}
c^{*} \simeq 0.00167 ; s^{*} \simeq 0.00168 \\
\zeta_{1} \simeq-0.0309 ; \zeta_{2} \simeq 0.0809 \\
\zeta_{3} \simeq-0.0307 ; \zeta_{4} \simeq 0.0807 .
\end{gathered}
$$

Using instead:

$$
a=2 ; \beta=\gamma=\frac{1}{2} ; \delta=\frac{1}{20} ; \eta=\frac{1}{30} ; \rho=\frac{1}{40} ; N=5
$$

we obtain:

$$
\begin{gathered}
c^{*} \simeq 0.00017 ; s^{*} \simeq 0.00006 \\
\zeta_{1} \simeq-0.0251 ; \zeta_{2} \simeq 0.0503 \\
\zeta_{3} \simeq-0.0188 ; \zeta_{4} \simeq 0.0440 .
\end{gathered}
$$

In general, repeating the same exercise for admissible parameter values, one can verify that the outcome is regularly $\zeta_{1}, \zeta_{3}<0$ while $\zeta_{2}, \zeta_{4}>0$. Therefore, the equilibrium is a saddle point. Accordingly, we may state our main result as follows: 
Proposition 7 The steady state equilibrium identified by $\left\{c^{*}(s), s^{*}(c), x^{*}=\delta, k^{*}=\eta\right\}$ is a saddlepoint.

\section{Concluding Remarks}

In this paper we have considered an optimal control model to study the dynamic behaviour of a multiproduct monopolist investing both in process innovation to reduce the marginal production cost and in product innovation to increase the degree of differentiation between any two varieties.

We have identified the conditions ensuring the existence of a unique steady state equilibrium, which is a saddle point. As for the economic features of the model, we have highlighted three results. First, we have found that firms face a higher incentive to invest both in process and in product innovation as the number of varieties increases. This is intuitively due to a cannibalization effect taking place among product varieties as the product space becomes progressively more crowded. Second, we have studied the composition on R\&D portfolio to understand which activity draws more resources. We have showed that, if the reservation price is sufficiently low, the firm finds it convenient to devote a larger amount of resources to process rather than product innovation irrespectively of the extent of the product range and the associated level of differentiation. If instead the reservation price is high enough, then the relative intensity of R\&D efforts is determined by the interaction between the number of varieties and the degree of differentiation, reflecting thus the cannibalization problem. Third, irrespectively of the demand level and the extension of the product range, we have proven that there always exists complementarity within the R\&D portfolio, i.e., any decrease in marginal production cost prompts for an analogous reduction of product substitutability, and conversely. This is true both along the optimal paths to the steady state and in steady state. 


\section{References}

[1] Abernathy, W. J. and J.M. Utterback (1975), A Dynamic Model of Process and Product Innovation, Omega, 3(6), 639-656.

[2] Abernathy, W. J. and J.M. Utterback (1982), Patterns of Industrial Innovation, in: M. L. Tushman and W. L. Moore, eds., Readings in the Management of Innovation, Boston: Pitman, 97-108.

[3] Adner R. and D. Levinthal (2001), Demand Heterogeneity and Technology Evolution: Implications for Product and Process Innovation, Management Science, 47(5), 611628.

[4] Amir, R. (1996), Cournot Oligopoly and the Theory of Supermodular Games, Games and Economic Behavior, 15, 132-148.

[5] Arrow, K. (1968), Applications of Control Theory to Economic Growth, in Dantzig, G.B. and A.F. Veinott, Jr (eds), Mathematics of the Decision Sciences, Part 2, Providence, American Mathematical Society.

[6] Athey, S. and A. Schmutzler (1995), Product and Process Flexibility in an Innovative Environment, RAND Journal of Economics, 26, 557-574.

[7] Bhattacharya, S. and D. Mookherjee (1986), Portfolio Choice in Research and Development, RAND Journal of Economics, 17, 594-605.

[8] Bonanno, G. and B. Haworth (1998), Intensity of Competition and the Choice between Product and Process Innovation, International Journal of Industrial Organization, 16, 495-510.

[9] Cellini, R., and L. Lambertini (2002), A Differential Game Approach to Investment in Product Differentiation, Journal of Economic Dynamics and Control, 27, 51-62. 
[10] Cellini, R. and L. Lambertini (2004), Private and Social Incentives Towards Investment in Product Differentiation, International Game Theory Review, 6, 493-508.

[11] Dasgupta, P. and E. Maskin (1987), The Simple Economics of Research Portfolios, Economic Journal, 97, 581-595.

[12] Doraszelski, U. (2004), Innovations, Improvements, and the Optimal Adoption of New Technologies, Journal of Economic Dynamics and Control, 28, 1461-1480.

[13] Harrington, J. E. (1995), Experimentation and Learning in a Differentiated Products Duopoly, Journal of Economic Theory, 77, 181-191.

[14] Klepper, S. (1996), Entry, Exit and Innovation over the Product Life Cycle, American Economic Review, 86, 562-583.

[15] Lambertini, L. (2003), The Monopolist's Optimal R\&D Portfolio, Oxford Economic Papers, 55, 561-578.

[16] Lambertini, L. (2004), Process and Product R\&D by a Multiproduct Monopolist: A Reply to Lin, Oxford Economic Papers, 56, 745-749.

[17] Lambertini, L. and R. Orsini (2000), Process and Product Innovation in a Vertically Differentiated Monopoly, Economics Letters, 68, 333-337.

[18] Lambertini, L. and G. Rossini (1998), Product Homogeneity as a Prisoner's Dilemma in a Duopoly with R\&D, Economics Letters, 58, 297-301.

[19] Lambertini, L., S. Poddar and D. Sasaki (1998), Standardization and the Stability of Collusion, Economics Letters, 58, 303-10.

[20] Lin, P. (2004), Process and Product R\&D by a Multiproduct Monopolist, Oxford Economic Papers, 56, 735-743. 
[21] Lin, P. and K. Saggi (2002), Product Differentiation, Process R\&D, and the Nature of Market Competition, European Economic Review, 46, 201-211.

[22] Mangasarian, O.L. (1966), Sufficient conditions for the Optimal Control of Nonlinear Systems, SIAM Journal of Control, 4, 139-152.

[23] Mantovani, A. (2005), Complementarity between product and process innovation in a monopoly setting, Economics of Innovation and New Technology, forthcoming.

[24] Milgrom, P. and J. Roberts (1990), Rationalizability, Learning, and Equilibrium in Games with Strategic Complementarities, Econometrica, 58, 1255-1278.

[25] Rosenkranz, S. (2003), Simultaneous Choice of Process and Product Innovation, Journal of Economic Behavior and Organization, 50, 183-201.

[26] Singh, N. and X. Vives (1984), Price and Quantity Competition in a Differentiated Duopoly, RAND Journal of Economics, 15, 546-554.

[27] Spence, M. (1976), Product Differentiation and Welfare, American Economic Review, $66,407-414$.

[28] Stalford, H. and G. Leitmann (1973), Sufficiency Conditions for Nash Equilibria in N-Person Differential Games, in Blaquiere, A. (ed.), Topics in Differential Games, Amsterdam, Elsevier.

[29] Vives, X. (1990), Nash Equilibrium with Strategic Complementarities, Journal of Mathematical Economics, 19, 305-321. 